\title{
Why are we still measuring red cell folate instead of just serum folate?
}

Tahir S. Pillay*1

\section{Nicholette M. Oosthuizen ${ }^{1}$}

\begin{abstract}
${ }^{1}$ Department of Chemical Pathology, Faculty of Health Sciences \& National Health Laboratory Service, University of Pretoria, Private Bag X1, Arcadia, Pretoria, South Africa

*Correspondence author:Department of Chemical Pathology, Faculty of Health Sciences \& National Health Laboratory Service, University of Pretoria, Private Bag X1, Arcadia, Pretoria, South Africa
\end{abstract}

Email: $\underline{\text { tspillay@gmail.com }}$

Tel: 012-319-2114; Fax: 012-328-3600

Keywords: serum folate, red cell folate, nutrition, vitamins

Folic acid or folate is a water-soluble vitamin from the B group and is involved in one-carbon metabolism. Folate deficiency or inadequate folate intake is associated with anaemia, neural tube defects and neuropsychiatric disorders. Furthermore, insufficient dietary intake of folate results in elevated plasma homocysteine, which is a risk factor for cardiovascular disease.

As with most other vitamins, the assessment of biological stores and intake is based on the concentration in body fluids. Studies of folate status have traditionally used serum/plasma or red blood cell folate. Both assays are widely available in laboratories. Red cell folate, favoured by many clinicians, is the more labour intensive test and therefore more costly than serum folate. Turnaround time is also longer because of the need to determine the haematocrit using a haematology cell counter followed by preparation of a haemolysate from the whole blood prior to running the assay. Factors related to the pre-treatment procedure, haematocrit and oxygenation of haemoglobin all contribute to analytical variability of red cell folate ${ }^{[1]}$ making coefficients of variation for red cell folate assays close to twice those of serum folate assays. The cost of red cell folate is between $40 \%$ and $55 \%$ higher than that of serum folate in the private sector and the South African National Health Laboratory Service (NHLS) respectively. We recently carried out a retrospective analysis of red cell and serum folate requests in the Tshwane metropolitan(Pretoria) area. Of the almost 21000 tests requested over an eight-year period, $2 \%$ demonstrated folate deficiency. This relatively low prevalence most likely reflects the success of the mandatory food fortification programme implemented in South Africa in October 2003 and is consistent with figures reported by studies conducted after introduction of fortification in other countries. ${ }^{[2]}$

A recent systematic review of serum folate and red cell folate revealed that red cell folate was subject to significant analytical variation and neither marker was superior in diagnosing folate deficiency ${ }^{[1]}$ However there was greater correlation of serum folate with homocysteine. Both increased with supplementation but serum folate showed the greater response to changes in dose. There is considerable difference in opinion nationally and internationally regarding the preferred sample. In the UK, the National Pathology Alliance benchmarking review recommended the measurement of serum folate. In Australia, red cell folate is the preferred sample. However, the available current evidence indicates the use of red blood cell folate is not justified clinically and analytically, given the higher costs. In an era where the costs of laboratory testing are increasingly being placed under the microscope, is it not time to dispense with the routine use of a test which is more expensive and 
subject to greater variation and therefore clinically less reliable? If we are intent on practising evidence-based laboratory medicine and reducing costs, then the answer is yes. However, there are a few caveats that readers should note: red cell folate is superior to serum folate in the following situations, after haemodialysis and between pregnancies as it gives a better assessment of folate status. Both markers perform equally well when assessing the risk of neural tube defect during pregnancy. Given the aforementioned issues and the importance of demand management in laboratory testing, it is clear that considerable savings could be achieved by changes in the testing of folate status.

\section{References}

1. Farrell CJ, Kirsch SH, Herrmann M. Red cell or serum folate: what to do in clinical practice? Clin Chem Lab Med 2013;51(3):555-569.[ http://dx.doi.org/10.1515/cclm-2012-0639][ PMID: 23449524]

2. Modjadji SEP, Alberts M, Mamabolo RL. Folate and iron status of South African nonpregnant rural women of childbearing age, before and after fortification of foods. S Afr J Clin Nutr 2007;20(3):89-93. 